\title{
Importance of Epigallocatechin and its Health Benefits
}

\author{
Mohamad Hesam Shahrajabian ${ }^{1}$, Wenli Sun ${ }^{1}$, Qi Cheng ${ }^{1,2,3, *}$
}

\section{Mohamad Hesam Shahrajabian', Wenli Sun', Qi Cheng ${ }^{1,2,3, *}$}

'Biotechnology Research Institute, Chinese Academy of Agricultural Sciences, Beijing, CHINA

${ }^{2}$ College of Life Sciences, Hebei Agricultural University, Baoding, Hebei, CHINA. ${ }^{3}$ Global Alliance of HeBAU-CLS\&HeQiS for BioAl-Manufacturing, Baoding, Hebei, CHINA.

\section{Correspondence}

\section{Prof. Qi Cheng}

${ }^{1}$ Biotechnology Research Institute, Chinese Academy of Agricultural Sciences, Beijing 100081, CHINA.

${ }^{2}$ College of Life Sciences, Hebei Agricultural University, Baoding-071000, Hebei, CHINA. Global Alliance of HeBAUCLS\&HeQiS for BioAl-Manufacturing, Baoding-071000, Hebei, CHINA.

Phone no: +86 13051039294

E-mail: chengqi@caas.cn

\section{History}

- Submission Date: 21-11-2020;

- Review completed: 02-12-2020;

- Accepted Date: 29-12-2020.

DOI : 10.5530/fra.2020.2.9

Article Available online

http://www.antiox.org

\section{Copyright}

(c) 2020 Phcog.Net. This is an openaccess article distributed under the terms of the Creative Commons Attribution 4.0 International license.

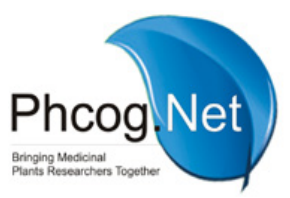

\begin{abstract}
Natural products have a wide range of diversity of multidimensional chemical structures which play a vital role which show the important nature as golden source for achieving the herbal drug discovery. Literature survey was accomplished using multiple databases including PubMed, Science Direct, ISI web of knowledge and Google Scholar. Epigallocatechin-3-gallate is the most abundant tea polyphenol, followed by other polyphenols, namely, catechin, epicatechin, epicatechin-3-gallate and epigallocatechin. The most important pharmacological activities of EGCG are antineoplastic, HIV infection, hypertension and associated complications, type II diabetes mellitus, its usage as cardioprotective, hepatoprotective, nephroprotective and its application in Alzheimer, Parkinson and Osteoporosis. Natural products have played a key role in drug discovery and development in modern days.
\end{abstract}

Key words: Natural Compounds, Medicinal Plants, Phloretin, Epigallocatechin.

\section{INTRODUCTION}

Traditional herbal medicines have been considered as a source of curative remedy, because chemical components of plants are used to promote health and prevent diseases ${ }^{1}$ and plants are invaluable sources of new drugs. ${ }^{1-13}$ Epigallocatechingallate (EGCG), which is also known as epigallocatechin-3-gallate, a type of catechin and it is the ster of epigallocatechin and gallic acid. ECGC is the most abundant in tea and used in many dietary supplements and beneficial to affect human health and disease. The aim of this mini-review article is survey on the most important pharmacological benefits of Epigallocatechin.

\section{EPIGALLOCATECHIN}

Epigallocatechin-3-gallate is the most abundant tea polyphenol, followed by other polyphenols, namely, catechin, epicatechin, epicatechin-3-gallate and epigallocatechin. ${ }^{14} \quad$ Epigallocatechingallate (EGCG), also known as epigallocatechin-3-gallate, is a polyphenolic flavonoid from tea (Camellia sinensis) possess various pharmacological activities such as anticancer, antimicrobial and antioxidant. ${ }^{15}$ Its neuroprotective effectagaint neural injuries and neurodegenerative diseses is also reported. ${ }^{16}$ It can also ameliorate protein and lipid damage induced by hepatotoxin, ethanol. ${ }^{17}$ EGCG carriers also appropriate for its wide application in food industry. ${ }^{18}$ The interaction mechanism of EGCG and natural $\alpha$-glucosidase (SCG) can be beneficial to the development of functional foods to prevent diabetes, ${ }^{19}$ or develop ative and environmental friendly packing materials for food industry. ${ }^{20}$ Its importance in cancer treatment is because of natural origin, safety and low cost, but EGCG challeng is its low bioavailability with various major limitations in EGCG studies which are study design, experimental bias, inconsistent results and reproductivity among different study cohorts. ${ }^{21}$ It can increase the potency of several chemotherapeutics such as doxorubicin, cisplating and tamoxifen, in vivo and in vitro, in many cancers, ${ }^{22}$ and a suitable adjuvant to potentiate anti-glioma therapies. ${ }^{23}$ EGCG may help in alleviating Methyl parathioninduced oocyte abnormalities. ${ }^{24} \mathrm{Wu}$ et al. ${ }^{25}$ showed that epicatechingallate is a potential inhibitor of a-amylase and a-glucosidase, which indicates its importance as a nutrient supplement for the prevention of diabetes mellitus. Its fatty acid derivatives use for the prevention and treatment of viral infections. ${ }^{26}$ Ling et al. ${ }^{27}$ introduced EGCG as a novel and safe chemopreventive agent for influenza A infection. EGCG has found appropriate as cryopreservation procedures in stallions with low-quality spern and possibly equine, to avoid or minimize DNA damages and preserve sperm plasma membrane integrity and mitochondrial activity. ${ }^{28}$ EGCG may improve growth performance and alleviate the oxidant damage by modulating the antioxidant properties of broilers. ${ }^{29}$ The most important pharmacolofical impacts of EGCG are presented in Table 1.

\section{CONCLUSION}

Epigallocatechinggallate is a teacatechin. The most important pharmacological activities of EGCG are antineoplastic, HIV infection, hypertension and associated complications, type II diabetes mellitus, 
Table 1: The most important pharmacological effects of EGCG.

EGCG Function

Autoxidation effect a. EGCG may lead to the formation of reactive oxygen species.

b. Its mechanism is based on the formation of EGCG quinone, EGCG dimer quinone and other related compounds and the formation of autoxidized products may contribute to the inhibition of fibrillation.

c. EGCG are found to form covalent adducts with cysteinylthiol residues in proteins through autoxidation to subsequently modulate protein function, which can be applied to treat human gastric cancer.

d. The stability and autoxidation of EGCG are realted to $\mathrm{pH}$, temperature, metal ion, antioxidant levels, oxygen levels, concentration of ECGC and other ingredients in tea.

Its effects for treatment of Alzheimers disease (AD), Parkinson's disease $(\mathrm{PD})$ and Huntington's disease (HD)

a. EGCG effects $\mathrm{AD}$ through oxidative stress, neurogenesis alteration and neuroinflammation.

b. EGCG suppressed the production of $A \beta$ and reduced inflammation, oxidative stress and cell apoptosis.

c. EGCG can also increase in the ey autophagy adaptor proteins NDP52 and p62.

d. EGCG reduce the A $\beta$ levels by enhancing andogenous APP proteolysis and decrease nuclear transloation of $\mathrm{c}-\mathrm{Abl}$.

e. EGCG induce an increase in the key autophay adaptor proteins NDP52 and P62.

f. EGCG reulates the iron-export ferroporting in substantianigra, reduce oxidative stress and exert a neurorescue effect against 1-methyl-4-phenyl-1,2,3,6-tetrahydropyridine (MPTP)-induced functional and neutochemical deficits. g. $\mathrm{AD}, \mathrm{PD}$ and $\mathrm{HD}$ are led by protein misfolding resulting from amyloid protein, which is a fibros polymer rich in $\beta$ sheets, formed by the self-assembly of proteins of different sequences, structures and functions.

Antitumor activity a. Introduction of 6-methoxycabonyl to Epigallocatechin-3-gallate (EGCG) is effective against gefitinib-resistant HCC827-Gef cells which can enhance its antitumor activities.

Antiviral activity a. It has antiviral acitivties for many viruses such as human immunodeficiency virus (HIV), herpes simplex virus (HSV), influenza virus (Flu) and hepatitis C virus (HCV) by inhibiting the entry the virus into the host cell.

b. EGCG can block the binding HIV-1 gp 120 to CD4 receptor and suppress the macrophage infiltration/activation in the rectal mucosa od macaques. Thus, EGCG can be considered as a novel, safe and cost-effective microbiocide for preventing sexual transmission of HIV-1.

Antifibrosis effect a. EGCG can inhibit the activation and proliferation of hepatic stellate cells and synthesis of collagen, in a rat model.

b. EGCG can reduce MMP-2 activity and possess the antibrosis effect via downregulation of the expresson of MMP-2 mRNA.

c. ECGCG can also inhibit the NF- $\mathrm{kB}$ to reverse the peritoneal fibrosis process.

Antimicrobial

a. Rinsing with EGCG solution may reduce the levels of mutans streptococci and lactobacilli in the oral cavity of activity children.

b. It may increase the DPPH radical scavenging activity and the enzyme inhibitory activity against $\alpha$-amylase and a-glucosidase which shows its antibacterial effects.

c. The application of EGCG may play important roles in cell behavior and beneficial for regenerative endodontic therapy due to the antibacterial cross-linking agent of EGCG and differentiation of human dental pulp cells (hDPCs) cultured in collagen scaffolds.

EGCC against a. It has fungicialacticities against Trichophyton mentagrophytes, T. rubrum, Cryptococcus neoformans and C. albicans.

b. It has activity against T. mentagrophytes.

c. EGCG synergistically increase the antigunfal potential of azola drugs which maybe helpful in preventing the development of drug resistance, in lowering the drug dosage and minimizing adverse effects.

a. EGCG has antimicrobial effects against bacteria causing food-borne disease, the most recognized bacterium are bacteria

$$
\text { Escherichia coli, Helicobacter pylori, Bacillus stearothermophilus, Clostridium thermoaceticum, Helicobacter pylori, }
$$
Salmonella typhi and Bacillus cereus.

EGC against viruses

a. HCV, HIV1, HBV, HSV-1/HSV-2, EBV, Adenovirus, Influenza virus, Enterovirus.

b. For HCV, inhibitory effect is viral entry by interference with binding to target cells.

c. For HIV-1, inhibitory effects are inhibition of integrase, inhibition of RT, destruction of virions by bidning to envelope and binding CD4 and interference with gp 120 binding.

d. For HBV, inhibitory effects are antigen expression, extracellular HBV DNA and cccDNA.

e. For HSV-1/HSV-2, inhibitory effects are damage and inactivation of virions probably by binding to envelope proteins.

f. For EBV, inhibitory effects are inhibition of transcription of immediate-early genes Rta, Zta and EA-D.

g. For Adenovirus, inhibitory effects are inactivation of virus pariticles, inhibition of intracellular virus growth and viral protease.

h. For influenza virus, inhibitory effects are alteration of physical integrity of virus particles, inhibiting of entry by binding to haemagglutinin.

i. For enterovirus, inhibitory effects are suppression of viral replication via modulation of cellular redox milieu. 
Anti-inflammatory a. EGCG may ameliorate OVA-induced airway inflammation by increasing the production of IL-10, the number of effect $\quad \mathrm{CD} 4{ }^{+} \mathrm{CD} 25^{+}{ }^{+}$oxp $3^{+}$Treg cells and expression of Foxp 3 mRNA in the lung tissue and it can be recommend for treating asthma.

b. ECGC inhibits the transfection of NF- $\mathrm{kB}$ and AP-1 to downregulate the expression of iNOS and COX-2 mainly by scavenging NO, peroxynitrite and other ROS/RNS and decreases the production of inflammatory factors to show the anti-inflammatory effects.

c. EGCG may inhibit species and it has been pointed out that ECGC may inhibit the IL-8 production of respiratory passge epithelium cell, which may reduce the severity of respiratory passge inflammatory response.

Anti-angiogenesis a. EGCG may inhbit tumor growth and angiogenesis which is possibly involved with the signaling intervention of MAPK/ERK1/2 and PI3K/AKT/HIF-1 $\alpha /$ VEGF pathways which is supposed to be a potential therapeutic reagent for anti-angiogensis treatment of solid tumors.

b. The modification of the 3"position methylation of EGCG (MethylEGCG) may reduce cell growth effects at a low concentration in vivo.

c. EGCG may prevent most of the IR-induced cellular and molecular events.

Antiobesity a. Epigallocatechingallate binds with human peroxisome proliferator-activated receptors gamma (PPAR) gamma at its active site and block its activity and this mode of action may be helpful for antiobesity development.

a. Peptide-conjugated formulations possess great cytotoxicity and great survivability and reduction in tumour volume

Its effects against in mice treated with peptide-conjugated shows EGCG as a novel drug delivery system in breat cancer therapy.

breast cancer a. EGCG may inhibit of ZIKV entery into the host cells

Anti Zika virus effect

Spermatogenesis a. EGCG at the concentration of $5 \mu \mathrm{M}$ has influence on hBMSCs. activity

its usage as cardioprotective, hepatoprotective, nephroprotective and its application in Alzheimer, Parkinson and Osteoporosis. Its importance in cancer treatment is because of natural origin, safety and low cost, but the main problem is its low bioavailability with various major limitations in EGCG studies.

\section{Funding}

This work was supported by the National Key R\&D Program of China (Research grant 2019YFA0904700)

\section{CONFLICT OF INTEREST}

The authors declare that they have no potential conflicts of interest.

\section{ABBREVIATIONS}

EGCG: Epigallocatechingallate; SCG: Natural a-glucosidase; AD: Alzheimer's disease; PD: Parkinson's disease; HD: Huntington's disease; MPTP: 1-methyl-4-phenyl-1,2,3,6-tetrahydropyridine; HIV: Human immunodeficiency virus; HSV: Herpes simplex virus; HCV: Hepatitis C virus; hDPCs: Human dental pulp cells; Methyl EGCG: Methylation of EGCG; PPAR: Peroxisome proliferator-activated receptors.

\section{REFERENCES}

1. Shahrajabian $M H$, Sun $W$, Cheng $\mathrm{Q}$. Chinese star anise (Illicium verum) and pyrethrum (Chrysanthemum cinerariifolium) as natural alternatives for organic farming and health care: A review. Aust J Crop Sci. 2020;14(03):517-23.

2. Shahrajabian $M H$, Sun $W$, Cheng $\mathrm{O}$. Traditional herbal medicine for the prevention and treatment of cold and flu in the autumn of 2020, overlapped with COVID-19. Nat Prod. Commun. 2020;15(8):1-10.

3. Shahrajabian $M H$, Sun $W$, Cheng $\mathrm{Q}$. Chinese onion (Allium chinense), an evergreen vegetable: A brief review. Pol J Agro. 2020;42:40-5.

4. Sun $W$, Shahrajabian $M H$, Cheng $\mathrm{O}$. Pyrethrum and organic and natural pesticide. J Biol Environ Sci. 2020;14(40):41-44.

5. Shahrajabian $\mathrm{MH}$, Sun $\mathrm{W}$, Cheng $\mathrm{Q}$. Considering white gold, cotton for its fiber, seed oil, traditional and modern health benefits. J Biol Environ Sci. 2020;14(40):25-39

6. Sun $\mathrm{W}$, Shahrajabian $\mathrm{MH}$, Cheng $\mathrm{Q}$. The insight and survey on medicinal properties and nutritive components of shallot. J Med Plant Res. 2019;13(18):452-7.

7. Shahrajabian $M H$, Sun $W$, Cheng $\mathrm{O}$. A review of astragalus species as foodstuffs, dietary supplements, a traditional Chinese medicine and a part of modern pharmaceutical science. Appl Ecol Environ Res. 2019;17(6):13371-82.

8. Shahrajabian MH, Sun W, Cheng Q. Tremendous health benefits and clinical aspects of Smilax china. Afr J Pharm Pharmacol. 2019;13(16):253-8.

9. Sun W, Shahrajabian MH, Cheng $\mathrm{Q}$. Anise (Pimpinella anisum I.), a dominant spice and traditional medicinal herb for both food and medicincal purposes. Cogent Biol. 2019;5(1673688):1-25.

10. Shahrajabian MH, Sun W, Cheng $\mathrm{Q}$. Chemical components and pharmacological benefits of Basil (Ocimum basilicum): A review. Int J Food Prop. 2020;23(1):1961 70 .

11. Shahrajabian $M H$, Sun $W$, Cheng $Q$. Clinical aspects and health benefits of ginger (Zingiber officinale) in both traditional Chinese medicine and modern industry. Acta Agric Sc and Sec B Soil Plant Sci. 2019;69(6):546-56.

12. Shahrajabian $M H$, Sun $W$, Cheng $Q$. A review of ginseng species in different regions as a multipurpose herb in traditional Chinese medicine, modern herbology and pharmacology science. J Med Plant Res. 2019;13(10):213-26.

13. Shahrajabian $M H$, Sun $W$, Shen $H$, Cheng $Q$. Chinese herbal medicine for SARS and SARS-CoV-2 treatment and prevention, encouraging using herbal medicine for COVID-19 outbreak. Agric Sc and Sec B Soil Plant Sci. 2020;70(5):437-43.

14. Tauber AL, Schweiker SS, Levonis SM. From tea to treatment: Epigallocatechin gallate and its potential involvement in minizing the metabolic changes in cancer. Nutr Res. 2018;74:23-36

15. Khalatbary AR, Khademi E. The green tea polyphenolic catechin epigallocatechin gallate and neuroprotection. Nutr Neurosci. 2020;23(4):281-94

16. Kaviarasan S, Sundarapandiyan R, Anuradha CV. Epigallocatechin gallate, a green tea phytochemical, attenuates alcohol-induced hepatic protein and lipid damage. Toxicol Mech Method. 2008;18(8):645-52.

17. Nilsuwan K, Guerrero P, Caba KDI, Benjakul S, Prodpran T. Properties and application of bilayer films based on poly (lactic acid) and fish gelatin containing epigallocatechin gallate fabricated by thermo-compression molding. Food Hydrocoll. 2020;105:105792.

18. DaiT, LiT, He X, Liu C, Chen J, McClements DJ. Analysis of inhibitory interaction between epigallocatechin gallate and alpha-glucosidase: A spectroscopy and molecular simulation study. Spectrochim Acta A. 2020;230:118023.

19. Ruan C, Zhang Y, Wang J, Sun $Y$, Gao X, Xiong G, et al. Preparation and antioxidant activity of sodium alginate and carboxymethyl cellulose edible films with epigallocatechin gallate. Int J Biol Macromol. 2019;134:1038-44.

20. Aggarwal V, Tuli HS, Tania M, Srivastava S, Ritzer EE, Pandey A, et al. Molecular mechanisms of action of epigallocatechin gallate in cancer: Recent trends and advancement. Semin Cancer Biol. 2020

21. Zhang W, Zhang W, Sun L, Xiang L, Lai X, Li Q, et al. The effects and mechanisms of epigallocateching-3-gallate on reversing multidrug resistance in cancer. Trends Food Sci Technol. 2019;93:221-33.

22. Le CT, Leenders WPJ, Molenaar RJ, VanNoorden CJF. Effects of the green tea polyphenol epigallocatechin-3-gallate on glioma: A critical evaluation of the literature. Nutr Cancer. 2018;70(3):317-33.

23. Hegde S, Poojary KK, Rasquinha R, Crasta DN, Gopalan D, Mutalin S, et al. Epigallocateching-3-gallate (EGCG) protects the oocytes from methyl parathion- 
induced cytoplasmic deformities by suppressing oxidative and endoplasmic reticulum stress. Pestic Biochem Phys. 2020;104588. DOI: 10.1016/j. pestbp. 2020.104588

24. Wu X, Hu M, Hu X, Ding H, Gong D, Zhang G. Inhibitory mechanism of epicatechingallate on $\alpha$-amylase and $\alpha$-glucosidase and its combinational effect with acarbose or epigallocatechinggallate. J Mol Liq. 2019;290:111202.

25. Kaihatsu K, Yamabe M, EbaraY. Antiviral mechanism of action of epigallocatechin3-O-gallate and its fatty acid esters. Molecules. 2018;23(10):2475.

26. Ling JX, Wei F, Li N, Li JJ, Chen LJ, Liu YY, et al. Amelioration of influenza virusinduced reactive oxygen species formation by epigallocatechin gallate derived from green tea. Acta Pharmacol Sin. 2012;33(12):1533-41.

27. Nouri H, Shojaeian K, Samadian F, Lee S, Kohram H, Lee IK. Using resveratrol and epigallocatechin-3-gallate to improve cryopreservation of stallion spermatozoa with low quality. J Equine Vet Sci. 2018;70:18-25

28. Abd El-Hack ME, Elnesr SS, Alagawany M, Gado A, Noreldin AE, Gabr AA. Impact of green tea (Camellia sinensis) and epigallocatechin gallate on poultry. World Poultry Sci J. 2020;76(1):49-63.

29. Tanaka T, Ishii T, Mizuno D, et al. (-)-Epigallocatechin-3-gallate suppresses growth of AZ521 human gastric cancer cells by targeting the DEAD-box RNA helicase p68. Free Radic Biol Med. 2011;50(10):1324-35.

30. Zhang ZX, Li YB, Zhao RP. Epigallocateching gallate attenuates $\beta$-Amyloid generation and oxidative stress involvement of PPAR $\gamma$ in N2a/APP695 cells. Neurochem Res. 2017;42(2):468-80.

31. Liu J, Zhong T, Yi P, Fan C, Zhang Z, Liang G, et al. A new epigallocatechingallate derivative isolated from Anhua dark tea sensitizes the chemosensitivity of gefitinib via the suppression of PI3K/mTOR and epithelial mesenchymal transition. Fitoterapia. 2020;143:104590.

32. Liu R, Zhang T, Wang T, Chang M, Jin Q, Wang X. Microwave-assisted synthesis and antioxidant activity of palmitoyl-epigallocatechingallate. LWT. 2020;101:6639.

33. Yasuda S, Horinaka M, Sakai T. Sulforaphane enhances apoptosis induced by Lactobacillus pentosus strain S-PT84 via TNF $\alpha$ pathway in human colon cancer cells. Oncol Lett. 2009;18(4):4253-61.

34. Kitamura $M$, Nishino $Y$, Obata $Y$, et al. Epigallocatechin gallate suppresses peritoneal fibrosis in mice. Chem Biol Interact. 2012;195(1):95-104

35. Vilela MM, Salvador SL, Teixeira IGL, DelArco MCG, DeRossi A. Efficacy of green tea and its extract, epigallocatechin-3-gallate, in the reduction of cariogenic microbiota in children: A randomized clinical trial. Archives Oral Biol. 2020;114:104727.

36. Evensen NA, Braun PC. The effects of tea polyphenols on Candida albicans: Inhibition of biofilm formation and proteasome inactivation. Can J Microbiol
2009;55(9):1033-9.

37. Behbahani JM, Irshad M, Shreaz $S$, Karched M. Synergistic effects of tea polyphenol epigallocatechin 3-O-gallate and azole drugs against oral Candida isolates. J Mycol Med. 2019;29(2):158-67.

38. Lee KM, Kim WS, Lim J, Nam S, Youn M, Nam SW, et al. Antipathogenic properties of green tea polyphenol epigallocatechingallate at concentrations below the MIC against enterohemorrhagic Escherichia coli O157: H7. J Food Prot. 2009;72(2):325-31.

39. Cui Y, Oh YJ, Lim J, Youn M, Lee I, Pak HK, et al. AFM study of the differential inhibitory effects of the green tea polyphenol (-)-epigallocatechin-3-gallate (EGCG) against gram-positive and gram-negative bacteria. Food Microbiol. 2012;29(1):80-7.

40. Isaacs C, Xu W, Merz G, Hillier S, Rohan L, Wen GY. Digallate dimmers of (-)-epigallocatechin gallate inactivate herpes simplex virus. Antimicrob Agents Chemother. 2011;55(12):5646-53.

41. Liu JB, Li JL, Zhuang K, Liu H, Wang X, Xiao QH, et al. Epigallocateching3-gallate local pre-exposure application prevents SHIV rectal infection of macaques. Mucosal Immunol. 2012;11(4):1230-8.

42. Shankar S, Marsh L, Srivastava RK. EGCG inhibits growth of human pancreatic tumors orthotopically implanted in Balb $\mathrm{C}$ nude mice through modulation of FKHRL1/FOXO3a and neuropilin. Mol Cell Biochem. 2013;372(1-2):83-94.

43. Liao ZH, Zhu HQ, Chen YY, Chen RL, Fu LX, Zhou H, et al. The epigallocatechin gallate derivative $Y_{6}$ inhibits human hepatocellular carcinoma by inhibiting

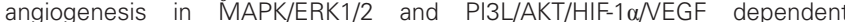
pathways. J Ethnopharmacol. 2020;259:112852

44. Sabetkar M, Low SY, Bradley NJ, Jacobs M, Naseem KM, Richard BK. The nitration of platelet vasodilator stimulated phosphoprotein following exposure to low concentrations of hydrogen peroxide. Platelets. 2008;19(4):282-92.

45. Tao F, Xiao C, Chen W, Zhang Y, Pan J, Jia Z. Covalent modification of $\beta$-lactoglobulin by (-)-epigallocatechin-3-gallate results in a novel antioxidant molecule. Int J Biol Macromol. 2019;126:1186-91.

46. Radhakrishnan R, Pooja D, Kulhari H, Gudem S, Ravuri HG, Bhargava S, et al. Bombesin conjugated solid lipid nanoparticles for improved delivery of epigallocatechingalate for breast cancer treatment. Chem Phys Lipids. 2019;224:104770.

47. Rodriguez $R$, Kondo $H$, Nyan $M$, et al. Implantation of green tea catechin $\alpha$-tricalcium phosphate combination enhances bone repair in rat skull defects. $J$ Biomed Mater Res B. 2011;98(2):263-71.

48. Jin $\mathrm{P}, \mathrm{Wu} \mathrm{H}, \mathrm{Xu} G$, Zheng I, Zhao J. Epigallocateching-3-gallate (EGCG) as a pro-osteogenic agent to enhance osteogenic differentiation of mesenchyma stem cells from human bone marrow: An in vitro study. Cell Tissue Res. 2014;356(2):381-90

\section{GRAPHICAL ABSTRACT}
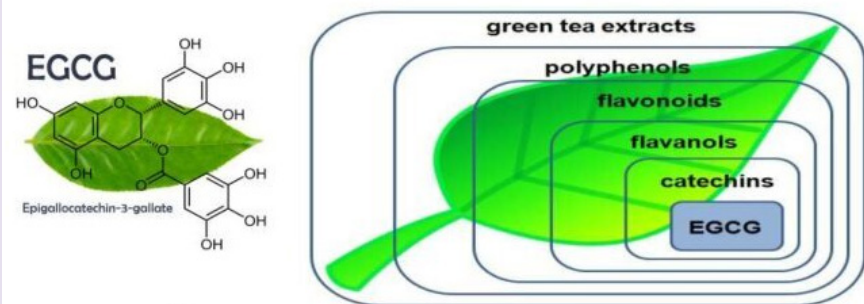

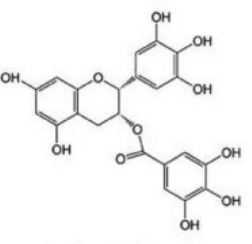

Epigallocatechin gallate (EGCG)

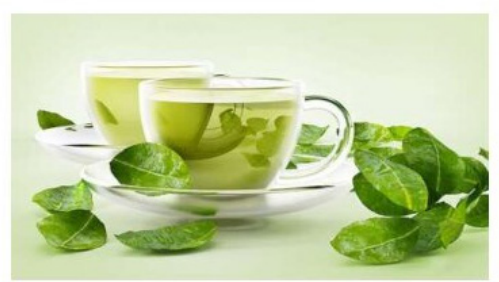

\section{SUMMARY}

- Epigallocatechinggallate is a tea catechin.

- The most important pharmacological activities of EGCG are antineoplastic, HIV infection, hypertension and associated complications, type II diabetes mellitus, its usage as cardioprotective, hepatoprotective, nephroprotective, and its application in Alzheimer, Parkinson, and Osteoporosis.

- Its importance in cancer treatment is because of natural origin, safety, and low cost, but the main problem is its low bioavailability with various major limitations in EGCG studies. 


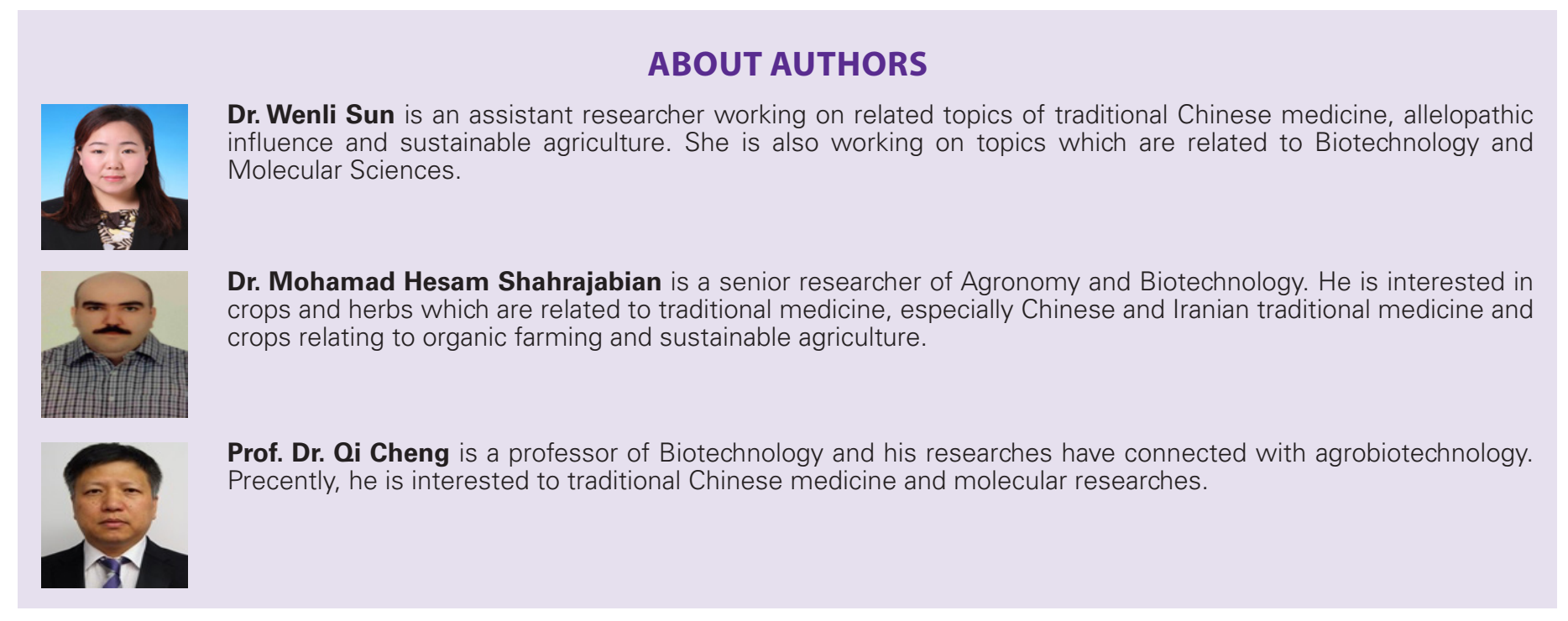

Cite this article: Shahrajabian M, Sun W, Cheng Q. Importance of Epigallocatechin and its Health Benefits. Free Radicals and Antioxidants. 2020;10(2):47-51. 